\title{
Assessment of Apical Foramen Position and Morphology in Maxillary Incisors Among South Indian Population: an in Vitro Study
}

\author{
Karthikeyan Gayathri ${ }^{1}$ and Raghu Sandhya ${ }^{2}$ \\ ${ }^{1}$ Saveetha Dental College and Hospitals, Saveetha Institute of Medical and \\ Technical Sciences (SIMATS), Saveetha University, Chennai 600077, India \\ ${ }^{2}$ Reader,Department of Conservative Dentistry and Endodontics, Saveetha Dental College and Hospitals, Saveetha \\ Institute of Medical and Technical Sciences (SIMATS), Saveetha University, Chennai 600077, India
}

\begin{abstract}
The aim of the study was to assess the apical foramen position and morphology in maxillary incisors in the south Indian population. This was an in vitro, experimental study in which 100 human permanent maxillary anterior teeth extracted for periodontal reasons from various patients in Chennai, India were studied. The extracted teeth consisted of 50 central incisors and 50 lateral incisors. All the specimens were observed under $3 \mathrm{X}$ magnification to assess the position and morphology of apical foramen.In the maxillary central incisors, the apical foramen was present in the range between 0.00 to $0.20 \mathrm{~mm}$ from the root apex in $66 \%, 0.20$ to $0.40 \mathrm{~mm}$ in $12 \%, 0.40$ to $0.60 \mathrm{~mm}$ in $8 \%, 0.60$ to $0.80 \mathrm{~mm}$ from the root apex in $14 \%$ of the teeth. In the lateral incisors the apical foramen was present in the range between 0.00 to $0.20 \mathrm{~mm}$ from the root apex in $60 \%, 0.20$ to $0.40 \mathrm{~mm}$ in $4 \%, 0.40$ to $0.60 \mathrm{~mm}$ in $20 \%, 0.60$ to $0.80 \mathrm{~mm}$ in $8 \%, 0.80$ to $1.00 \mathrm{~mm}$ from the root apex in $8 \%$ of the teeth. The shape of the apical foramen in maxillary central incisors was round (76\%), oval $(16 \%)$ and irregular $(8 \%)$. In lateral incisors the apical foramen shape was round $(78 \%)$, oval (14\%), irregular (6\%), and elliptical (2\%). Chi-square test was performed to see the association between the distance of the apical foramen from the root apex and variation in the shape of the apical foramen from the root in maxillary central and lateral incisors. The results obtained were statistically significant with the p-value of 0.00 for both. In most cases in the maxillary incisor teeth the apical foramen coincided with the anatomic apex. The most common shape of the apical foramen was observed to be round shape. There is also a statistical significance present between the shape of the apical foramen and the distance of the apical foramen from the root apex in both maxillary central and lateral incisors.
\end{abstract}

KEY WORDS: APICAL FORAMEN POSITION, APICAL FORAMEN MORPHOLOGY, ROOT APEX, APICAL CONSTRICTION.

\section{INTRODUCTION}

For a successful endodontic treatment to be carried out a thorough knowledge about the root canal anatomy

\section{ARTICLE INFORMATION}

*Corresponding Author: drsandhyaendo@gmail.com Received 10th June 2020 Accepted after revision 2nd August 2020 Print ISSN: 0974-6455 Online ISSN: 2321-4007 CODEN: BBRCBA

Thomson Reuters ISI Web of Science Clarivate Analytics USA and Crossref Indexed Journal

\section{Clarivate}

NAAS Journal Score 2020 (4.31) SJIF: 2020 (7.728)

A Society of Science and Nature Publication,

Bhopal India 2020. All rights reserved.

Online Contents Available at: http//www.bbrc.in/

Doi: http://dx.doi.org/10.21786/bbrc/13.7/47 and the apical region of the root is necessary. The apical region of the tooth comprises significant landmarks which marks the extent for root canal instrumentation and filling. Earlier studies regarding the root canal anatomy are most commonly reported fromAmerican, Ugandan, Turkish, Sudanese, Caucasian, Srilankan, and Chinese population (Grove, 1926). Starting from the Caucasians to the Africans and Asians, the patterns of root canal system follow a racial characteristic making endodontic management extremely challenging to the clinical practitioners (Zaatar et al., 1997) Various 
methods to investigate the root canal anatomy have been identified.

The terminal portion of a tooth root exhibits four distinct landmarks; the apical constriction(AC), apical foramen $(\mathrm{AF})$, roots apex (anatomic and radiographic), and cement junction (CDJ). AF is an important anatomical landmark which is the major apical opening of the root canal (Simon, 1994).The anatomic apex (AA) is the root end that is identified morphologically (Akers, 2010)Deviation of AF from the root apex is common, with a reported frequency of 17\%-100\% (Martos et al., 2009).

Review of literature showed that studies pertaining to root canal morphology of maxillary anterior teeth among Indian population is very limited. On the contrary, root canal anatomy of premolars and molars in Indian population has been extensively investigated. Further studies pertaining to root apical anatomy were even limited. Literature review reveals that the AF of maxillary and mandibular canines in Indian population exhibited variations in their locations and on average AC was $0.89 \mathrm{~mm}$ coronal to AA (Amardeep, Raghu and Natanasabapathy, 2014) (Dummer, McGinn and Rees, 1984).

We have numerous highly cited publications on well designed clinical trials and lab studies (Govindaraju, Neelakantan and Gutmann, 2017; Azeem and Sureshbabu, 2018; Jenarthanan and Subbarao, 2018; Manohar and Sharma, 2018; Nandakumar and Nasim, 2018; Teja, Ramesh and Priya, 2018; Janani and Sandhya, 2019; Khandelwal and Palanivelu, 2019; Malli Sureshbabu et al., 2019; Poorni, Srinivasan and Nivedhitha, 2019; Rajakeerthi and Ms, 2019; Rajendran et al., 2019; Ramarao and Sathyanarayanan, 2019; Siddique and Nivedhitha, 2019; Siddique et al., 2019; Siddique, Nivedhitha and Jacob, 2019). This has provided the right platforms for us to pursue the current study. The aim of the study was to assess apical foramen position and morphology in maxillary incisors among south Indian population.

\section{MATERIAL AND METHODS}

This was an in vitro, experimental study in which 100 human permanent maxillary anterior teeth extracted for periodontal reasons from various patients in Chennai, India were studied. The extracted teeth consisted of 50 central incisors and 50 lateral incisors. Teeth with deep caries, cracks, fracture, incompletely formed root apices and those with root canal fillings were excluded from the study. All teeth were cleaned free of attached calculus and soft tissue using ultrasonic scalers and curette and were then preserved in 10\% formalin until use.The storage and handling of the teeth were performed as per the centers for disease control and prevention guidelines and regulation. All the specimens were numbered from 1 to 50 for the central incisors and from 51 to 100 for the lateral incisors. All the specimens were then subjected to manual observation with the naked eye followed by observation under a SLR camera (model: Nikon D3500) at
3X magnification to assess the position and morphology of apical foramen. Photographs were taken of each specimen. A Vernier caliper was used to measure the distance of the AF from the AA. The values obtained were calculated. Descriptive statistical analysis was done.

Graph 1: Bar graph shows the distribution of distance of the apical foramen from the root apex in maxillary central incisors (X-axis represents distance in $\mathrm{mm}, \mathrm{Y}$-axis represents the number of teeth). It was noted that the most common distance present in maxillary central incisors was between $0.00-0.20 \mathrm{~mm}$ ( 66.66\%).

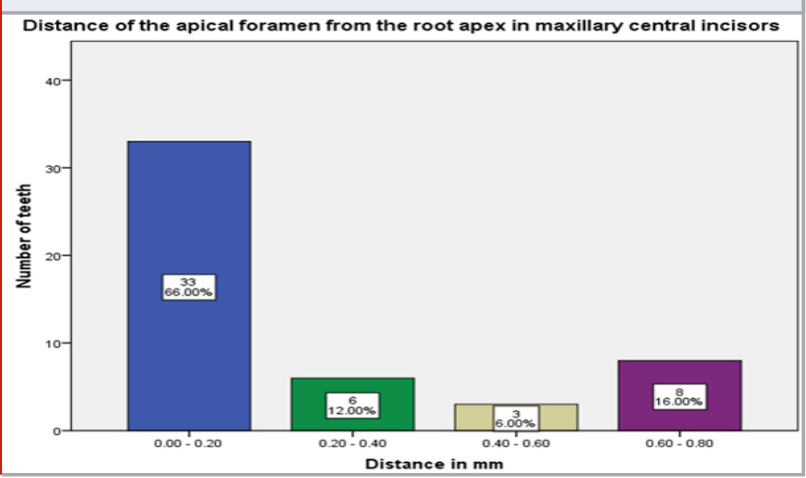

Graph 2: Bar graph shows the distribution of distance of the apical foramen from the root apex in maxillary lateral incisors (X-axis represents distance in $\mathrm{mm}, \mathrm{Y}$-axis represents the number of teeth). It was noted that the most common distance present in maxillary lateral incisors was between $0.00-0.20 \mathrm{~mm}(60.00 \%)$.

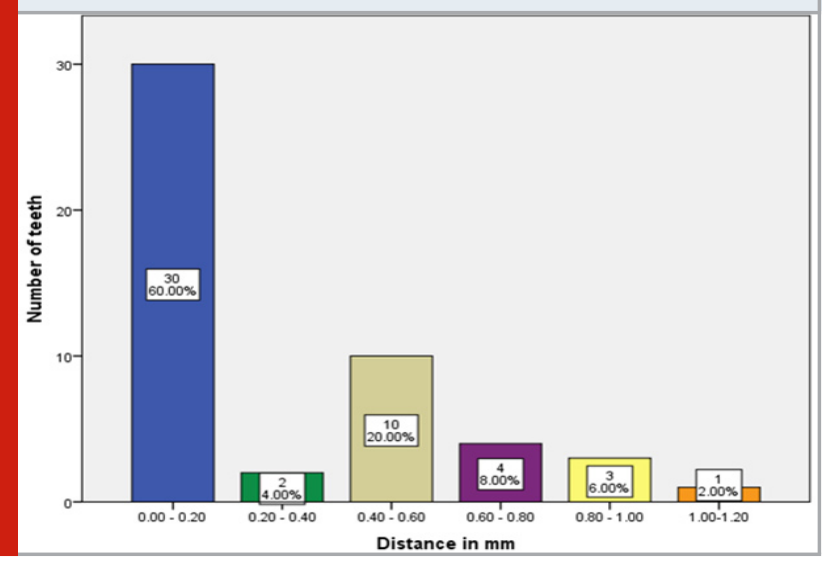

\section{RESULTS AND DISCUSSION}

In the maxillary central incisors, the apical foramen was present in the range between 0.00 to $0.20 \mathrm{~mm}$ from the root apex in $66 \%, 0.20$ to $0.40 \mathrm{~mm}$ in $12 \%, 0.40$ to $0.60 \mathrm{~mm}$ in $8 \%, 0.60$ to $0.80 \mathrm{~mm}$ from the root apex in $14 \%$ of the teeth. In the lateral incisors the apical foramen was present in the range between 0.00 to $0.20 \mathrm{~mm}$ from the root apex in $60 \%, 0.20$ to $0.40 \mathrm{~mm}$ in $4 \%, 0.40$ to $0.60 \mathrm{~mm}$ in $20 \%, 0.60$ to $0.80 \mathrm{~mm}$ in $8 \%, 0.80$ to $1.00 \mathrm{~mm}$ from the root apex in $8 \%$ of the teeth. The shape of the apical foramen in maxillary central incisors was round (76\%), oval (16\%) and irregular (8\%). In lateral incisors 
the apical foramen shape was round (78\%), oval (14\%), irregular (6\%), and elliptical (2\%). (Graph 1,2,3,4, Figure $1,2)$. Chi-square test was performed to see the association between the distance of the apical foramen from the root apex and variation in the shape of the apical foramen from the root in maxillary central and lateral incisors. The results obtained was statistically significant with the p-value of 0.00 for both $($ Graph 5,6)

Graph 3: Bar graph shows variation in the shape of the apical foramen from the root in maxillary central incisors (X-axis represents shape variation, $\mathrm{Y}$-axis represents the number of teeth). It was noted that the most common shape variation in maxillary central incisors was round ( 76.00\%).

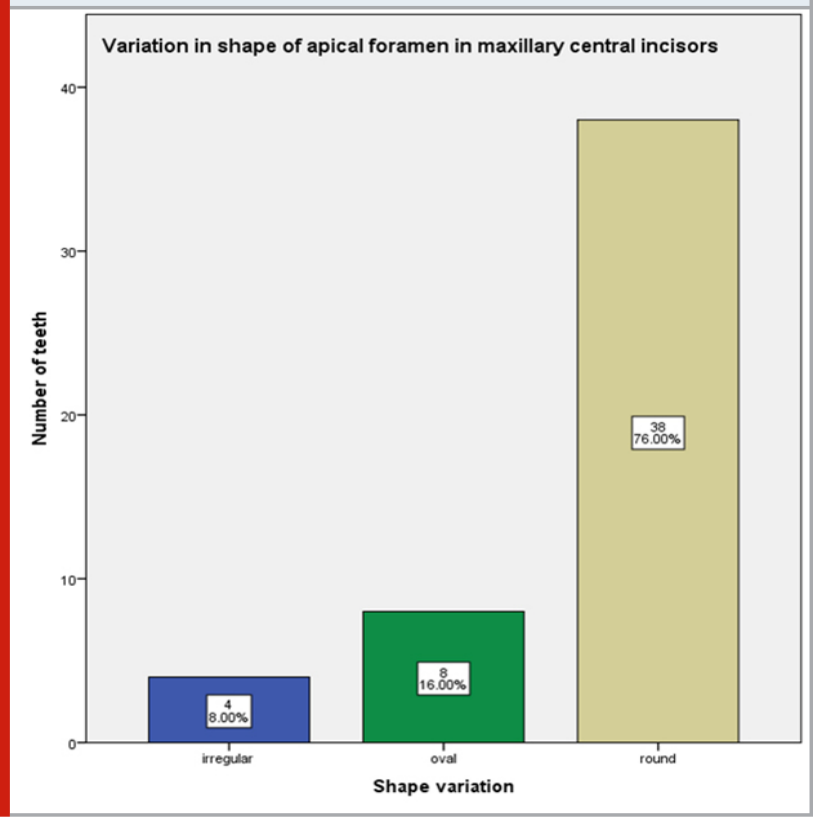

Graph 4: Bar graph shows variation in the shape of the apical foramen from the root in maxillary lateral incisors (X-axis represents shape variation, $\mathrm{Y}$-axis represents the number of teeth). It was noted that the most common shape variation in maxillary lateral incisors was round ( $78.00 \%)$.

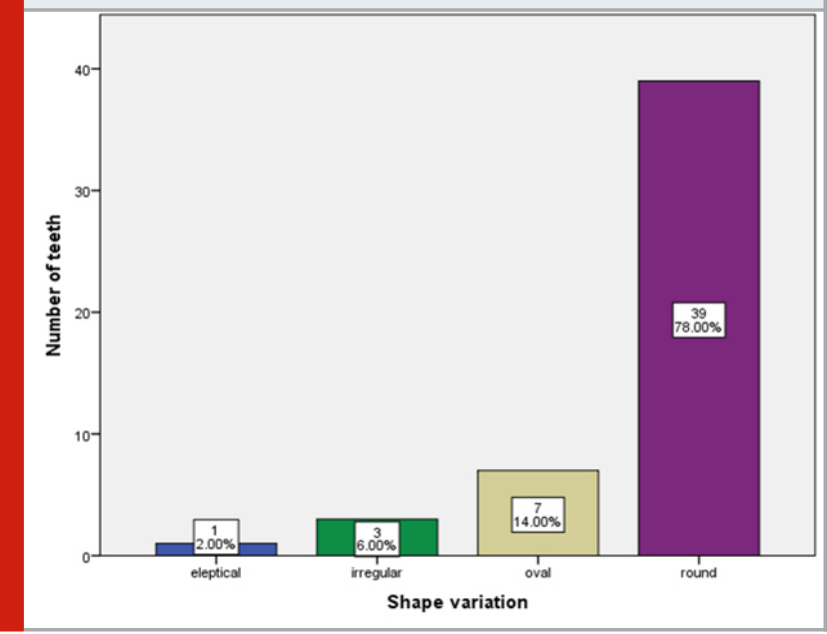

Graph 5: Bar graph shows the association between the distance of the apical foramen from the root apex and variation in the shape of the apical foramen from the root in maxillary central incisors (X-axis distance in $\mathrm{mm}$, Y-axis represents variation of shape of social foramen). It was noted that the most common shape present between the distance of 0.00-0.20 was round, most common shape present between the distance of $0.20-0.40$ was oval, most common shape present between the distance of 0.40-0.60 was round and most common shape present between the distance of 0.60-0.80 was irregular and oval. Chi-square test was done and $p$-value was $0.00(p=<0.05)$ hence statistically significant proving that there is an association present between shape of the apical foramen and distance of the apical foramen from the root apex in maxillary central incisors

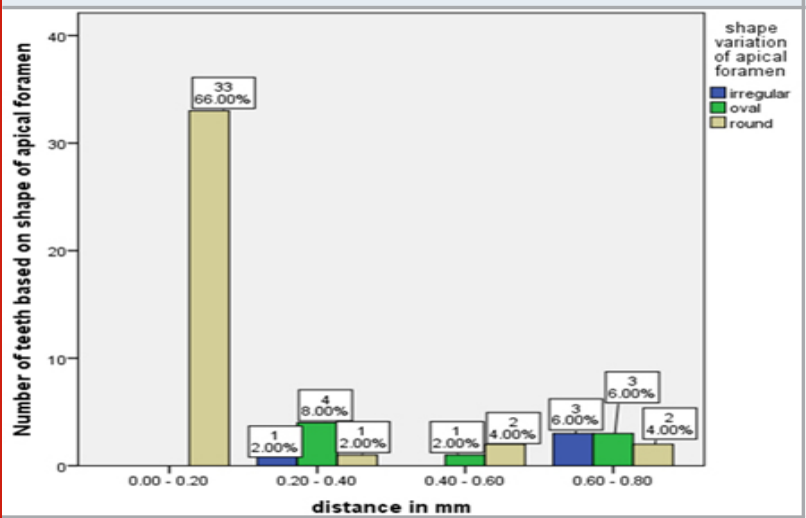

The morphology of root canal anatomy constituting apical exit of the canal, apical deltas, lateral canals, intercanal anastomosis, and canal designs with their complexities itself, provide conducive environments for microbial colonization resulting in periapical infections. Successful elimination of these microbes and debris from these complexities plays a major influence in the treatment outcome(Vertucci, 2005). It becomes the responsibility of the endodontist to disinfect the canal of these microbes. Hence, a proper knowledge of the root canal anatomy and apical root anatomy will only facilitate the clinicians to achieve this goal, thereby resulting in a drastic reduction in posttreatment failure rates.

As mentioned by a study conducted by Ghasemi et al., the complexity of the root canal system is influenced by genetics, and this factor should always be considered before interpreting and comparing the results of various other morphological studies(Ghasemi et al., 2017). Numerous techniques have been used for assessing the presence of apical and lateral foramina in extracted teeth such as sectioning of teeth, infiltration of the root canal system with dyes, radiographic techniques, scanning electron microscopy, and dental computerized tomography(Chapman, 1969) (Kartal and Yanıko囚lu, 1992; Gutierrez and Aguayo, 1995; Scarfe, Fana and Farman, 1995). 
Graph 6: Bar graph shows the association between the distance of the apical foramen from the root apex in maxillary lateral incisors and variation in the shape of the apical foramen from the root in maxillary lateral incisors (X-axis distance in $\mathrm{mm}, \mathrm{Y}$-axis represents variation of shape of social foramen). It was noted that the most common shape present between the distance of 0.00-0.20 $\mathrm{mm}$ was round, most common shape present between the distance of $0.20-0.40 \mathrm{~mm}$ was round, most common shape present between the distance of $0.40-0.60 \mathrm{~mm}$ was round, most common shape present between the distance of $0.60-0.80 \mathrm{~mm}$ was irregular and oval, most common shape present between the distance of $0.80-1.00 \mathrm{~mm}$ was irregular and most common shape present between the distance of $0.60-1.00 \mathrm{~mm}$ was elliptical. Chi-square test was done and $\mathrm{p}$-value was $0.00(\mathrm{p}=<0.05)$ hence statistically significant proving that there is an association present between shape of the apical foramen and distance of the apical foramen from the root apex in maxillary lateral incisors

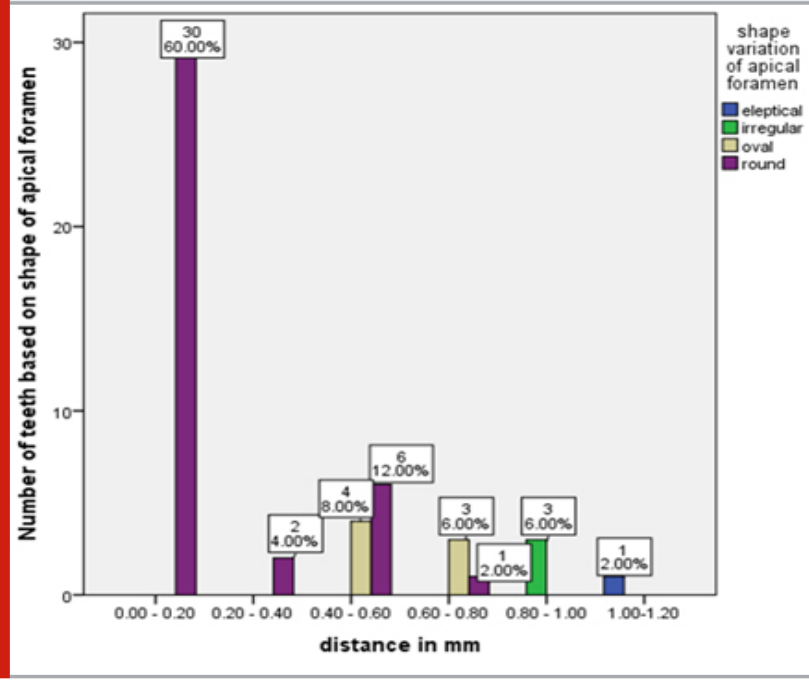

Figure 1: Maxillary central incisor apical foramen having (a) round shape, (b) oval shape, (c) irregular shape

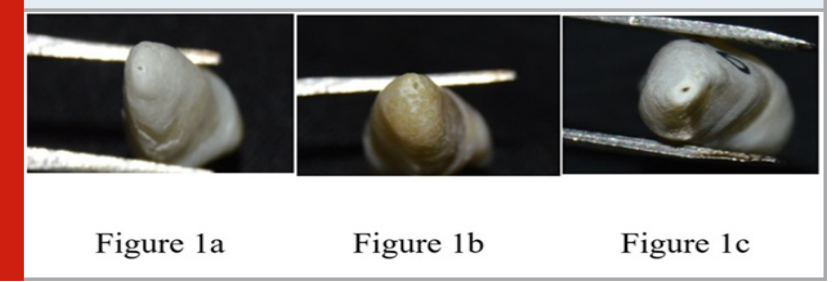

Figure 2: Maxillary lateral incisor apical foramen having (a) round shape, (b) oval shape, (c) irregular shape, (d) elliptical shape

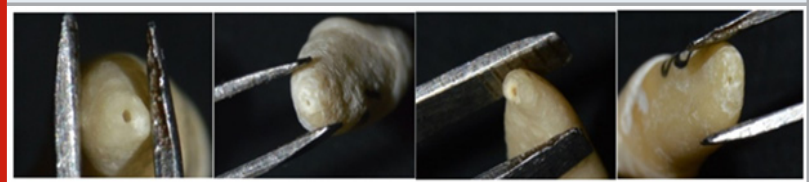

Figure 2a Figure 2b Figure 2c Figure 2d
Other methods employed for assessing the root canal anatomy in extracted teeth include contrast mediumenhanced radiographic techniques, modified canal staining and clearing technique and surgical operating microscopy (Altman et al., 1970; Fan et al., 2008; Weng et al., 2009)

In the present population studied, the position of AF in maxillary anterior teeth was determined with the aid of an SLR camera. SLR cameras emerge as a tool that offers many benefits such as, magnification and aids with proper documentation. The usage of documentation for medico-legal, insurance, patient communication, and lecturing purposes, as well as for communication with staff or colleagues, is also a major benefit. In the present study the position of the apical foramen coincided with the root apex in $66 \%$ of the cases in the maxillary central incisors. In case of the lateral incisors the apical foramen coincided with root apex in $60 \%$ of the cases.

This was in accordance with a study conducted by Martos et al in the Brazilian population, where the location of the major foramen was in the center of the root apex in $58.4 \%$ of specimens(Martos et al., 2010)The results of the study conducted by Teo et al.in a population in Singapore showed 54.3 percent with the foramen coincident with the root apex in maxillary anterior which was also consistent with the results obtained from this study. The deviation of the apical foramen from anatomical apex was $60.25 \%$ in the frontal teeth in a study conducted by Martic et al which was in contradiction with the result from this study. Studies have reported that only 1\%-5\% of maxillary central incisors had their AF at a distance of $>1 \mathrm{~mm}$ from the apex of the root, whereas a higher frequency of AF deviation in posterior teeth has been observed which was consistent with our study as well (Marti冈 et al., 1998)

The morphology of the apical foramen showed a predominance of the rounded shape followed by the oval shape in a study conducted by Martos et al. which was consistent with our study was well(Martos et al., 2009) Conducting this study will help with improving and modifying the endodontic procedure according to the population. Being aware of the variations in the apical foramen shape helps us to determine the type of obturating material that can generally be used for a good apical seal.

\section{CONCLUSION}

From the results obtained the apical foramen was found to be in the range between 0.00 to $0.20 \mathrm{~mm}$ from the root apex in most of the cases of the maxillary central incisors and maxillary lateral incisor. Most common apical foramen shape was found to be round in maxillary central incisor and maxillary lateral incisor. There is also a statistical significance present between the shape of the apical foramen and the distance of the apical foramen from the root apex in both maxillary central and lateral incisors. 
Conflict of Interest: The author would like to declare there was no conflict of interest

\section{ACKNOWLEDGEMENTS}

I would like to thank my esteemed institution for providing me with the opportunity to do this study and would also like to thank my guide for her expert advice and encouragement which helped me with the completion of this study.

\section{REFERENCES}

Akers, H. F. (2010) 'AN ENDODONTIC GLOSSARY OF TERMS', Australian Endodontic Newsletter, pp. 9-9. doi: 10.1111/j.1747-4477.1985.tb00130.x.

Altman, M. et al. (1970) 'Apical root canal anatomy of human maxillary central incisors', Oral Surgery, Oral Medicine, Oral Pathology, pp. 694-699. doi: 10.1016/0030-4220(70)90396-8.

Amardeep, N. S., Raghu, S. and Natanasabapathy, V. (2014) 'Root Canal Morphology of Permanent Maxillary and Mandibular Canines in Indian Population Using Cone Beam Computed Tomography', Anatomy Research International, pp. 1-7. doi: 10.1155/2014/731859.

Azeem, R. A. and Sureshbabu, N. M. (2018) 'Clinical performance of direct versus indirect composite restorations in posterior teeth: A systematic review', Journal of conservative dentistry: JCD, 21(1), pp. 2-9. Chapman, C. E. (1969) 'A Microscopic Study of the Apical Region of Human Anterior Teeth', International Endodontic Journal, pp. 52-58. doi: 10.1111/j.13652591.1969.tb01227.x.

Dummer, P. M., McGinn, J. H. and Rees, D. G. (1984) 'The position and topography of the apical canal constriction and apical foramen', International endodontic journal, 17(4), pp. 192-198.

Fan, B. et al. (2008) 'Identification of a C-shaped canal system in mandibular second molars-part II: the effect of bone image superimposition and intraradicular contrast medium on radiograph interpretation', Journal of endodontia, 34(2), pp. 160-165.

Ghasemi, N. et al. (2017) 'A Review on Root Anatomy and Canal Configuration of the Maxillary Second Molars', Iranian endodontic journal, 12(1), pp. 1-9.

Govindaraju, L., Neelakantan, P. and Gutmann, J. L. (2017) 'Effect of root canal irrigating solutions on the compressive strength of tricalcium silicate cements', Clinical oral investigations, 21(2), pp. 567-571.

Grove, C. J. (1926) 'Faulty Technic in Investigations of the Apices of Pulpless Teeth', The Journal of the American Dental Association (1922), pp. 746-747. doi: 10.14219/jada.archive.1926.0137.

Gutierrez, J. H. and Aguayo, P. (1995) 'Apical foraminal openings in human teeth. Number and location', Oral surgery, oral medicine, oral pathology, oral radiology, and endodontics, 79(6), pp. 769-777.

Janani, K. and Sandhya, R. (2019) 'A survey on skills for cone beam computed tomography interpretation among endodontists for endodontic treatment procedure', Indian journal of dental research: official publication of Indian Society for Dental Research, 30(6), pp. 834-838.

Jenarthanan, S. and Subbarao, C. (2018) 'Comparative evaluation of the efficacy of diclofenac sodium administered using different delivery routes in the management of endodontic pain: A randomized controlled clinical trial', Journal of conservative dentistry: JCD, 21(3), pp. 297-301.

Kartal, N. and Yanıko冈lu, F. Ç. (1992) 'Root canal morphology of mandibular incisors', Journal of Endodontics, pp. 562-564. doi: 10.1016/s00992399(06)81215-X.

Khandelwal, A. and Palanivelu, A. (2019) 'Correlation Between Dental Caries And Salivary Albumin In Adult Population In Chennai: An In Vivo Study', Brazilian Dental Science, 22(2), pp. 228-233.

Malli Sureshbabu, N. et al. (2019) 'Concentrated Growth Factors as an Ingenious Biomaterial in Regeneration of Bony Defects after Periapical Surgery: A Report of Two Cases', Case reports in dentistry, 2019, p. 7046203.

Manohar, M. P. and Sharma, S. (2018) 'A survey of the knowledge, attitude, and awareness about the principal choice of intracanal medicaments among the general dental practitioners and nonendodontic specialists', Indian journal of dental research: official publication of Indian Society for Dental Research, 29(6), pp. 716-720.

Marti区, D. et al. (1998) 'Morphometrical analysis of main and accessory canals in apical root portion of frontal teeth', Collegium antropologicum, 22 Suppl, pp. 153-159.

Martos, J. et al. (2009) 'Topographical evaluation of the major apical foramen in permanent human teeth', International Endodontic Journal, pp. 329-334. doi: 10.1111/j.1365-2591.2008.01513.x.

Martos, J. et al. (2010) 'Morphologic analysis of the root apex in human teeth', Journal of endodontia, 36(4), pp. 664-667.

Nandakumar, M. and Nasim, I. (2018) 'Comparative evaluation of grape seed and cranberry extracts in preventing enamel erosion: An optical emission spectrometric analysis', Journal of conservative dentistry: JCD, 21(5), pp. 516-520.

Poorni, S., Srinivasan, M. R. and Nivedhitha, M. S. (2019) 'Probiotic strains in caries prevention: A systematic review', Journal of conservative dentistry: JCD, 22(2), pp. 123-128. 
Rajakeerthi, R. and Ms, N. (2019) 'Natural Product as the Storage medium for an avulsed tooth - A Systematic Review', Cumhuriyet Dental Journal, 22(2), pp. 249256.

Rajendran, R. et al. (2019) 'Comparative Evaluation of Remineralizing Potential of a Paste Containing Bioactive Glass and a Topical Cream Containing Casein Phosphopeptide-Amorphous Calcium Phosphate: An in Vitro Study', Pesquisa brasileira em odontopediatria e clinica integrada, 19(1), pp. 1-10.

Ramarao, S. and Sathyanarayanan, U. (2019) 'CRA Grid - A preliminary development and calibration of a paper-based objectivization of caries risk assessment in undergraduate dental education', Journal of conservative dentistry: JCD, 22(2), pp. 185-190.

Scarfe, W. C., Fana, C. R., Jr and Farman, A. G. (1995) 'Radiographic detection of accessory/lateral canals: use of RadioVisioGraphy and Hypaque', Journal of endodontia, 21(4), pp. 185-190.

Siddique, R. et al. (2019) 'Qualitative and quantitative analysis of precipitate formation following interaction of chlorhexidine with sodium hypochlorite, neem, and tulsi', Journal of conservative dentistry: JCD, 22(1), pp. 40-47.

Siddique, R. and Nivedhitha, M. S. (2019) 'Effectiveness of rotary and reciprocating systems on microbial reduction: A systematic review', Journal of conservative dentistry: JCD, 22(2), pp. 114-122.

Siddique, R., Nivedhitha, M. S. and Jacob, B. (2019) 'Quantitative analysis for detection of toxic elements in various irrigants, their combination (precipitate), and para-chloroaniline: An inductively coupled plasma mass spectrometry study', Journal of conservative dentistry: JCD, 22(4), pp. 344-350.

Simon, J. H. (1994) 'The apex: how critical is it?', General dentistry, 42(4), pp. 330-334.

Teja, K. V., Ramesh, S. and Priya, V. (2018) 'Regulation of matrix metalloproteinase-3 gene expression in inflammation: A molecular study', Journal of conservative dentistry: JCD, 21(6), pp. 592-596.

Vertucci, F. J. (2005) 'Root canal morphology and its relationship to endodontic procedures', Endodontic Topics, pp. 3-29. doi: 10.1111/j.16011546.2005.00129.x.

Weng, X.-L. et al. (2009) 'Root Canal Morphology of Permanent Maxillary Teeth in the Han Nationality in Chinese Guanzhong Area: A New Modified Root Canal Staining Technique', Journal of Endodontics, pp. 651-656. doi: 10.1016/j.joen.2009.02.010.

Zaatar, E. I. et al. (1997) 'Frequency of endodontic treatment in Kuwait: radiographic evaluation of 846 endodontically treated teeth', Journal of endodontia, 23(7), pp. 453-456. 